\title{
Drug Rash With Eosinophilia and Systemic Symptoms Syndrome Induced by Allopurınol
}

\author{
Mukaddes Kavala ${ }^{\mathrm{a}}$, Ayse Serap Karadag ${ }^{\mathrm{a}}$, Filiz Topaloglu Demir ${ }^{\mathrm{a}, \mathrm{c}}$, Ilkin Zindancia, \\ Zafer Turkoglua ${ }^{\text {a }}$ Berkant Oman ${ }^{\mathrm{a}}$, Ebru Zemheri ${ }^{\mathrm{b}}$, Emin Ozlu ${ }^{\mathrm{a}}$
}

\begin{abstract}
Drug rash with eosinophilia and systemic symptoms (DRESS) syndrome is an adverse drug reaction caused by an apparent group of drugs which can cause $10-20 \%$ mortality. It is characterized by a latency period ranging from 3 weeks to 3 months after the introduction of the offending drug. The syndrome is defined by the presence of fever, rash, eosinophilia, atypical lymphocytes and multiorgan involvement. We present a 39-year-old woman who developed fever, nausea, a pruritic erythematous maculopapular rash and facial edema during her sixth week of the treatment with allopurinol as a case of DRESS syndrome. Diagnosis was confirmed by the drug rash, eosinophilia and systemic involvement including adenopathy, toxic hepatitis and pericardial effusion. Allopurinol was discontinued and intravenous prednisolone $60 \mathrm{mg}$ /day was started. The patient's clinical appearance and eosinophilia improved within first 2 days. Awareness of hematologic abnormalities and systemic involvement along with drug rush, by physicians is critical for early diagnosis of this life-threatening syndrome.
\end{abstract}

Keywords: DRESS syndrome; Allopurinol; Drug reactions

\section{Introduction}

Drug rash with eosinophilia and systemic symptoms (DRESS) syndrome, previously named "drug hypersensitivity syndrome" is severe adverse drug-induced reaction. Diagnosis of DRESS syndrome is challenging, because the pat-

\footnotetext{
Manuscript accepted for publication May 19, 2014

${ }^{a}$ Department of Dermatology, S.B Istanbul Medeniyet University, Goztepe Training and Research Hospital, Istanbul, Turkey

${ }^{b}$ Department of Pathology, S.B Istanbul Medeniyet University, Goztepe Training and Research Hospital, Istanbul, Turkey

${ }^{\mathrm{c}}$ Corresponding Author: Filiz Topaloglu Demir, Department of

Dermatology, S.B Istanbul Medeniyet University, Goztepe Training and Research Hospital, Istanbul, Turkey. Email: filizsvet@yahoo.com

doi: http://dx.doi.org/10.14740/jmc1811w
}

tern of cutaneous eruption and the types of organs involved are various. We describe a case of DRESS syndrome induced by allopurinol, and emphasize the importance of heterogeneity of clinical presentation which often results in initial misdiagnosis.

\section{Case Report}

A 39-year-old woman with a history of fever, malaise, pharyngitis and diffuse maculopapular rash on her body was admitted to the infection diseases department. She was started on amoxicillin/clavulanic acid treatment for a suspected upper respiratory tract infection. Five days after starting the previously mentioned antibiotic, she was consulted to us and transferred to our dermatology department. Her medical history was notable for appendectomy and pituitary gland tumor operation 3 and 5 years ago, and she had been treated with allopurinol for increased uric acid levels. Seven weeks after the initiation of allopurinol she had developed a skin eruption which began on the trunk and upper limbs and gradually disseminated for a couple of days. Physical examination revealed a generalized maculopapular exanthema, some of them with edematous follicular accentuation, periorbital edema and generalized lymphadenopathy (Fig. 1a, b). The patient was febrile $\left(38.2{ }^{\circ} \mathrm{C}\right)$.

Laboratory findings showed a WBC count of $14 \times 10^{9} / \mathrm{L}$ with $7.6 \%$ eosinophils. Liver function tests were abnormal with increased levels of AST (455 U/L, N: 0 - $35 \mathrm{U} / \mathrm{L})$, ALT (565 U/L, N: 0 - 35 U/L), GGT (532 U/L, N: 0 - 50 $\mathrm{U} / \mathrm{L}$ ) and alkaline phophatase (346 IU/L, N: $30-120 \mathrm{U} / \mathrm{L}$ ). There was an increase in serum creatinine $(1.64 \mathrm{mg} / \mathrm{dL}, \mathrm{N}$ : $0.50-9 \mathrm{mg} / \mathrm{dL})$ and urea $(89 \mathrm{mg} / \mathrm{dL}, \mathrm{N}: 17-43 \mathrm{mg} / \mathrm{dL})$ and decreased creatinine clearance $(61.72 \mathrm{~mL} / \mathrm{min}, \mathrm{N}: 85-125$ $\mathrm{mL} / \mathrm{min}$ ). Blood cultures, urine and stool cultures and nasalthroat swabs were negative for bacteria, protozoa or helminthic eggs. Serologic tests including syphilis, EBV, HIV and hepatitis $\mathrm{B}$ and $\mathrm{C}$ and antinuclear antibody were negative. Chest radiograhy was normal. Echocardiography showed pericardial effusion $0.8 \mathrm{~cm}$ around left ventricle and abdominal sonography showed moderate hepatomegaly. The skin biopsy yielded mild acanthosis and leukocytoclastic vasculi- 

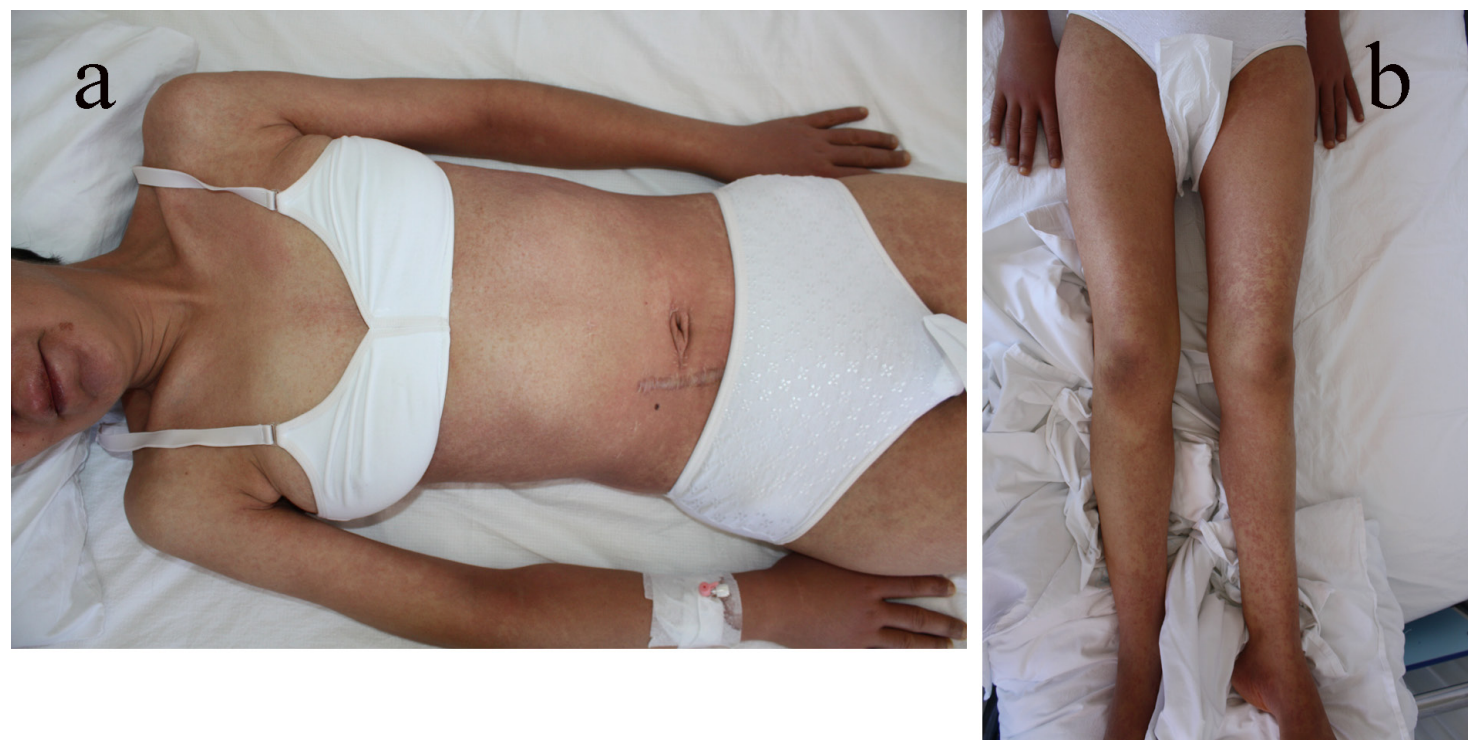

Figure 1. (a) A generalized maculopapular exanthema on the trunk and upper extremities. (b) A maculopapular exanthema on lower extremities.

tis in the upper dermis (Fig. 2). Allopurinol was immediately withdrawn, and systemic prednisolone $75 \mathrm{mg}$ daily and antihistamines were initiated. The patient's clinical appearance and laboratory abnormalities began to improve. The dosage of corticosteroids was gradually tapered and stopped as the clinical and laboratory abnormalities resolved over 6 months in order to prevent a recurrence of the symptoms of the disease.

\section{Discussion}

DRESS syndrome is a potentially life-threatening syndrome including a severe skin eruption, fever, hematologic abnormalities and internal organ involvement. The mortality rate has been estimated at $10 \%[1,2]$. DRESS syndrome most

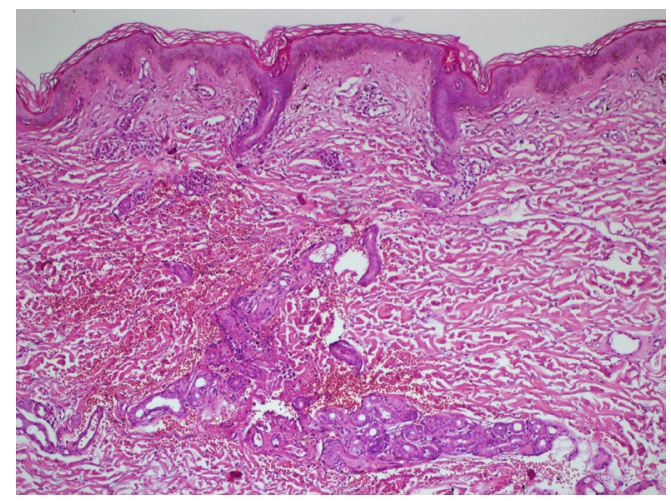

Figure 2. H\&E $(\times 20)$. Mild acanthosis and leucocythoclastic vasculitis in the upper dermis. commonly arises 3 - 8 weeks after exposure to the culprit drug. Our patient presented to the infection disease department 6 weeks after initiation of allopurinol. Fever often up to $38-40{ }^{\circ} \mathrm{C}$ and rash is the first sign seen in $90-100 \%$ and $90 \%$ of cases respectively [3]. The face, trunk and upper extremities are initially affected and the eruption is usually macular erythema that becomes confluent. Swelling of the face, with a marked periorbital involvement is a warning to the diagnosis, occurring in about $25 \%$ of patients [4]. Local or generalized lymphadenopathy is found in $70-75 \%$ of cases [5]. Various hematologic abnormalities are observed including leukocytosis, eosinophilia and atypical lymphocytes similar to mononucleosis. Liver involvement is the most common visceral manifestation, after the lymphadenopathy. Hepatitis with elevation of liver transaminases is found in $51 \%$ of cases [2]. Renal involvement is also reported in patients, being particularly evident in cases due to allopurinol [6]. Our patient's constellation of symptoms and course were consistent with the natural progression of DRESS syndrome. Severe organ involvement may develop, such as fulminant hepatitis, eosinophilic interstitial lung disease, interstitial nephropathy, pericarditis, myocarditis and pancreatitis, 1 - 2 weeks after skin eruption [1,2].

The aromatic anticonvulsants, salazosulfapyridine, dapsone and minocycline are frequently associated with DRESS syndrome, but a variety of other drugs can induce this syndrome [1]. Although the pathogenesis is unclear, different mechanisms have been implicated involving detoxification defects, immunological reactions, slow acetylation and reactivation of human herpes virus 6 [5-8].

Therapeutic approach consists of immediate discontinuation of the offending drug and administration of systemic 
corticosteroids, as the first choice. Intravenous immunoglobulins, plasmapheresis or a combination of these therapies can also be used if the symptoms get worse [4].

A correct and early diagnosis is necessary as DRESS syndrome is potentially associated with serious and sometimes fatal visceral involvement.

\section{Conflict of Interest}

There are no conflicts of interest among all authors regarding this article.

\section{References}

1. Tas S, Simonart T. Management of drug rash with eosinophilia and systemic symptoms (DRESS syndrome): an update. Dermatology. 2003;206(4):353-356.

2. Michel F, Navellou JC, Ferraud D, Toussirot E, Wendling D. DRESS syndrome in a patient on sulfasalazine for rheumatoid arthritis. Joint Bone Spine. 2005;72(1):8285.

3. Bocquet H, Bagot M, Roujeau JC. Drug-induced pseu- dolymphoma and drug hypersensitivity syndrome (Drug Rash with Eosinophilia and Systemic Symptoms: DRESS). Semin Cutan Med Surg. 1996;15(4):250-257.

4. Shear NH, Spielberg SP. Anticonvulsant hypersensitivity syndrome. In vitro assessment of risk. J Clin Invest. 1988;82(6):1826-1832.

5. Descamps V, Valance A, Edlinger C, Fillet AM, Grossin M, Lebrun-Vignes B, Belaich S, et al. Association of human herpesvirus 6 infection with drug reaction with eosinophilia and systemic symptoms. Arch Dermatol. 2001;137(3):301-304.

6. Picard D, Janela B, Descamps V, D'Incan M, Courville $\mathrm{P}$, Jacquot S, Rogez S, et al. Drug reaction with eosinophilia and systemic symptoms (DRESS): a multiorgan antiviral T cell response. Sci Transl Med. 2010;2(46):4662.

7. Shiohara T, Inaoka M, Kano Y. Drug-induced hypersensitivity syndrome (DIHS): a reaction induced by a complex interplay among herpesviruses and antiviral and antidrug immune responses. Allergol Int. 2006;55(1):1-8.

8. Kito Y, Ito T, Tokura Y, Hashizume H. High-dose intravenous immunoglobulin monotherapy for drug-induced hypersensitivity syndrome. Acta Derm Venereol. 2012;92(1):100-101. 